\title{
Gravity inversion by means of growing bodies
}

\author{
Antonio G. Camacho $\geq$, Fuensanta G. Montesinos $\geq$, \\ and Ricardo Vieira $\geq$
}

\section{ABSTRACT}

This paper presents a gravity inversion method for determining the volumes of bodies with pre-established density contrasts. The method works step-by-step on a prismatic partition of the subsurface volume, expanding the anomalous bodies to fit the observed gravity values in a systematic exploration of model possibilities. The process is treated in a 3-D context; at the same time, it can determine a simple regional trend. Moreover, positive and negative density contrasts are simultaneously accepted. The solution is obtained by a double condition: (1) the $\ell_{2}$-fitness to the observed gravity data (model fitness) and (2) the minimization of the total (weighted) anomalous mass (model smoothness). A positive parameter is used to balance the two minimization terms. The method is applied to a simulated example and also to a real example: the volcanic island of Gran Canaria (Canary Islands, Spain). In both cases, the results obtained show the possibilities of the method.

\section{INTRODUCTION}

The inverse gravimetric problem, namely the determination of a subsurface mass density distribution corresponding to an observed gravity anomaly, has an intrinsic nonuniqueness in its solution (e.g., Al-Chalabi, 1971). Moreover, data must be considered as insufficient and inaccurate. Nevertheless, particular solutions can be obtained by including additional information about the model parameters (subsurface structure) and about the data parameters (statistical properties of the inexact data, e.g., Gaussian distribution). The inversion methods vary in accordance with the amount of information about the subsurface structure (from very precise geological information to light mathematical conditions) and, mainly, with the kind of model parameters selected (geometrical parameters or densities).

For the gravity inversion methods which consider the densities of the elements of a regular subsoil partition as unknowns (with nondiscrete values), the linear approach seems to be a better choice (e.g., Camacho et al., 1997). In this case, the solution fits the observed data very well, but its geometrical properties are somewhat diffuse because of the rather smooth variation of the resulting anomalous densities. Otherwise, the methods which look for the geometrical properties of anomalous bodies with fixed density contrast (e.g., Pedersen, 1979; Barbosa et al., 1997) correspond to a nonlinear context and offer interesting results, limited to the validity of the hypothesis used.

Generally, the usual inversion methods look for analytical solutions by means of linear approaches or iterative methods for linearizable problems. Unfortunately, linearized techniques depend strongly on the accuracy of initial estimates of the model parameters (Rothman, 1985). For the fully nonlinear treatment, the methods of exploration of the model space often give the best option (Tarantola, 1988). This exploration process can be conducted randomly (Silva and Hohmann, 1983) or systematically.

We present an inversion method that can be included in this last group of systematic exploration. A strong hypothesis is adopted: the subsurface anomalous structure is characterized by prescribed mass density contrasts. Therefore, the problem consists of determining the geometry of the anomalous volumes corresponding to those density contrasts. For that, the subsurface volume is divided into a fixed discrete 3-D partition of prismatic elements, and the anomalous volumes are constructed by means of an "expansion approach." In this sense, our proposal is related to the "bubbling" method of Zidarov (1990) and the "open-reject-fill" method of René (1986).

As opposed to Zidarov's (1990) approach, in our approach step-by-step, prismatic elements are selected by means of systematic testing of each possibilities of model growth, and added to the existing elements. In his paper, René (1986) developed a 2-D expansion approach by using a "maternal" structure formed by square "seeds" that grow by incorporating only contiguous elements. The René method does not require additional hypotheses but uses only positive (or only negative) density contrasts, and models with both positive and

Published on Geophysics Online October 5, 1999. Manuscript received by the Editor March 9, 1998 ; Revised manuscript received May 25, 1999. ¿Ciudad Universitaria, Instituto de Astronomía y Geodesía, Facultad de CC. Matemáticas, 28040 Madrid, Spain. E-mail: camacho@eucmax.sim. ucm.es; fuensant@eucmax.sim.ucm.es or fuen@iagmat1.mat.ucm.es; vieira@eucmax.sim.ucm.es or vieira@iagmat1.mat.ucm.es.

$£ 2000$ Society of Exploration Geophysicists. All rights reserved. 
negative anomalies are not possible. To relax these limitations, we present an approach with the following new features: (1) we suppose a 3-D context, (2) nongridded nonplanar inaccurate data are accepted, (3) a "maternal" structure is not required, (4) a simple regional trend can also be simultaneously determined, (5) matter expansion can appear everywhere (not only for contiguous elements), (6) not requiring "contiguous" expansion lets us consider nonregular subsoil partitions (e.g., with deeper blocks bigger than shallow blocks), (7) if previous qualified models exist, they can be incorporated and, above all, (8) positive and negative density contrasts are simultaneously accepted. The last two improvements are obtained by means of an additional condition to the model.

\section{INVERSION METHOD}

Let us consider $n$ gravity stations $P_{i}\left(x_{i}, y_{i}, z_{i}\right), i=1, \ldots, n$, not necessarily gridded, located on a rugged topography and with observed anomalous gravity values $g_{i}$. We suppose a Gaussian distribution for the observation uncertainties given by a covariance matrix $\mathbf{Q}_{\mathbf{D}}$ (as deduced from a data analysis). The subsurface volume close to the survey area is broken down into a discrete 3-D partition of $m$ prismatic elements. The size and resolution of this partition are related to the gravity stations distribution (diameter and step) and, also, are conditioned by the capacity of the computer. The gravity attraction, $A_{i j}$, at the $i$ th station $P_{i}\left(x_{i}, y_{i}, z_{i}\right)$, due to the $j$ th prism, for unit density, is given by Pick et al. (1973):

$$
\begin{aligned}
& A_{i j}=\geq G\left[\left[(x \ln ) y+\left(x^{2}+y^{2}+z^{2}\right)^{\frac{1}{2}}\right)\right. \\
& \left.+y \ln ) x+\left(x^{2}+y^{2}+z^{2}\right)^{\frac{1}{2}}\right) \\
& \left.\left.\left.\left.+z \arctan ) z\left(x^{2}+y^{2}+z^{2}\right)^{\frac{1}{2}} x \geq 1 y^{\geq 1}\right)\right]_{u_{1}^{j} \geq x_{i}}^{u_{2}^{j} \geq x_{i}}\right]_{v_{1}^{j} \geq y_{i}}^{v_{2}^{j} \geq y_{i}}\right]_{w_{1}^{j} \geq z_{i}}^{w_{2}^{j} \geq z_{i}},
\end{aligned}
$$

where $G$ is the gravitation constant, the edges of the $j$ th prism are parallel to the reference axes, and the limiting coordinates for its volume are $u_{1}^{j}, u_{2}^{j}$ for the $x$ coordinate, $v_{1}^{j}, v_{2}^{j}$ for the $y$ coordinate, and $w_{1}^{j}, w_{2}^{j}$ for the $z$ coordinate. The matrix, $\mathbf{A}$, with components $A_{i j}$, is the design matrix of the physical configuration of the problem and contains the effect of the rugged terrain, the station distribution, subsoil partition, etc.

We look for a gravity inversion using a step-by-step matter expansion approach based on a systematic exploration of the growth possibilities upon a prismatic partition. First, if previous information about the model structure is known (which is not necessary), it can be initially incorporated. We suppose this information to be expressed as initial values $\rho_{j}^{0}, j=1, \ldots, m$, for the prism densities and a corresponding covariance matrix $\mathbf{Q}_{\mathbf{M}}$ corresponding to the previous model confidence (Tarantola, 1988). For a problem without previous information about the model structure, we can adopt $\rho_{j}^{0}, j=1, \ldots, m$, and take a model covariance matrix $\mathbf{Q}_{\mathbf{M}}$ given by a diagonal normalizing matrix of nonnull elements that are the same as the diagonal elements of $\mathbf{A}^{T} \mathbf{Q}_{\mathbf{D}}^{\geq 1} \mathbf{A}$. The elements of this matrix can be used also to limit the whole subsurface volume to study, bounding the accepted model uncertainty. The gravity values calculated for this initial density structure at points $P_{i}, i=1, \ldots, n$, are

$$
g_{i}^{0}=\sum_{j=1}^{m} A_{i j} \rho_{j}^{0} .
$$

After a step-by-step approach, the final model, $\rho_{j}, j=$ $1, \ldots, m$, will be obtained by adding to the initial values $\rho_{j}^{0}$ an additional density contrast $\Delta \rho_{j}$. These incremental values are constrained by a strong hypothesis: the adjusted anomalous density contrast $\Delta \rho_{j}$ of the prismatic elements which are modified can have only two local values-a previously prescribed positive value ("increased" prisms) or a previously prescribed negative value ("decreased" prisms). For the "unchanged" prisms, we can suppose a null value for the corresponding $\Delta \rho_{j}$. Here, we note that the prescribed incremental values (positive and/or negative) can be fixed with different values for different regions of the tested subsurface volume (local values). This hypothesis gives us a model of unchanged/increased/decreased prisms which fits the observed anomalies and which gives us interesting geometrical information (to the extent that the initial hypothesis is valid). The method works step-by-step by modifying the densities of the prisms (with positive or negative prescribed values) so that the modeled gravity anomalies fit (with a scale factor $f \geq 1$ ) the observed gravity anomalies. The anomalous mass grows (by means of increasing volume) while its corresponding gravity field remains proportional to the observed one. Moreover, a simple (e.g., linear) regional trend can be simultaneously adjusted. This process is shown below by means of an arbitrary step of the growth approach.

For an arbitrary $(k+1)$ th step, $k$ prisms have been definitively modified (with the positive or negative fixed additional values) and the modeled gravity values will be

$$
g_{i}^{c}=g_{i}^{0}+\sum_{j=\ell_{1}}^{\ell_{k}} A_{i j} \Delta \rho_{j},
$$

where $j=\ell_{1}, \ldots, \ell_{k}$ are the indexes corresponding to the previously modified prisms with densities $\rho_{j}^{0}+\Delta \rho_{j}$. Now, we look for one new prism to modify throughout the $m-k$ unchanged prisms. For the $j$ th unchanged prism, $j \neq \ell_{1}, \ldots, \ell_{k}$, the following equation system can be considered:

$$
\begin{gathered}
\left.\left.g_{i} \geq\right) g_{i}^{c}+A_{i j} \Delta \rho_{j}\right) f \geq\left(p_{0}+p_{x}\left(x_{i} \geq x_{M}\right)\right. \\
\left.+p_{y}\left(y_{i} \geq y_{M}\right)\right)=v_{i}, \quad i=1, \ldots, n,
\end{gathered}
$$

where $x_{i}, y_{i}$ are the planar coordinates of the $i$ th station; $x_{M}, y_{M}$ are the coordinates of an arbitrary central point for the survey (for example, mean coordinates); $f \geq 1$ is an unknown scale factor for fitting the "actual" model anomalies $\left(g_{i}^{c}+A_{i j} \Delta \rho_{j}\right)$ to the observed ones, $g_{i}$; and, $p_{0}, p_{x}, p_{y}$ are three unknown values which adjust a regional trend (a 1-degree polynomial surface, for simplicity of the further formulation). The positive and the negative prescribed values are successively tested for the additional density contrast $\Delta \rho_{j}$ as follows.

The acceptation of positive and negative values and the inclusion of the trend unknowns give us a nonuniqueness problem if only a minimization criterion for the residuals is used. To solve that, an additional condition of model variation minimization can be used. Then, the unknown parameters $f, p_{0}, p_{x}, p_{y}$ are adjusted for a mixed minimization criteria as

$$
\mathbf{v}^{T} \mathbf{Q}_{D}^{\geq 1} \mathbf{v}+\rho f^{2} \mathbf{m}^{T} \mathbf{Q}_{M}^{\geq 1} \mathbf{m}=\min .,
$$


where $\mathbf{v}=\left(v_{1}, \ldots, v_{n}\right)^{T}$ ( $T$ for transposed $)$ as defined by equation (1), $\rho$ is a positive factor fixed (see below) for balance between model fitness and model smoothness, and

$$
\left.\mathbf{m}=) \Delta \rho_{\ell_{1}}, \ldots, \Delta \rho_{\ell_{k}}, \Delta \rho_{j}\right)^{T} .
$$

The first term of the minimization functional (2) corresponds to the fit residues weighted with the data quality matrix. The second term is a weighted addition of the model densities. Nevertheless, taking into account the fact that the covariance matrix $\mathbf{Q}_{M}^{\geq 1}$ contain the prism volumes as factors, this second term is connected with the anomalous mass of the model.

The solutions of the equation system (1) and (2) can be calculated for the adopted prism and $\Delta \rho_{j}$ as

$$
\begin{aligned}
f & =\left(s_{r g} \geq p_{0} s_{r u} \geq p_{x} s_{r x} \geq p_{y} s_{r y}\right) /\left(s_{r r}+\rho s_{m m}\right), \\
p_{0} & =\left(F_{u g} \geq p_{x} F_{u x} \geq p_{y} F_{u y}\right) / F_{u u}, \\
p_{x} & =\left(G_{x g} \geq p_{y} G_{x y}\right) / G_{x x}, \\
p_{y} & =\left(G_{x x} G_{y g} \geq G_{x y} G_{x g}\right) /\left(G_{y y} G_{x x} \geq G_{x y} G_{x y}\right),
\end{aligned}
$$

where the subscripts $r, g, u, x$, and $y$ refer to the $n$-vectors $\mathbf{r}, \mathbf{g}$, $\mathbf{u}, \mathbf{x}$, and $\mathbf{y}$ with the following $i$ th components:

$$
\begin{gathered}
(\mathbf{x})_{i}=x_{i} \geq x_{M}, \quad(\mathbf{y})_{i}=y_{i} \geq y_{M}, \quad(\mathbf{u})_{i}=1, \\
(\mathbf{r})_{i}=g_{i}^{c}+A_{i j} \Delta \rho_{j}, \quad(\mathbf{g})_{i}=g_{i},
\end{gathered}
$$

and where $s_{m m}=\mathbf{m}^{T} \mathbf{Q}_{M}^{\geq 1} \mathbf{m}$, and the coefficients $s_{r g}, s_{r u}, s_{r x}, s_{r y}$, $s_{r r}, s_{m m}, F_{u g}, F_{u x}, F_{u y}, F_{u u}, G_{x g}, G_{x y}, G_{x x}, G_{y g}$, and $G_{y y}$ are calculated with the subsequent expressions, replacing the subscripts $a$ and $b$ by the corresponding pairs of subscripts $r, g, u, x, y$ :

$$
\begin{gathered}
s_{a b}=\mathbf{a}^{T} \mathbf{Q}_{D}^{\geq 1} \mathbf{b}, \quad F_{a b}=s_{a b}\left(s_{r r}+\rho s_{m m}\right) \geq s_{a r} s_{b r}, \\
G_{a b}=F_{u u} F_{a b} \geq F_{u a} F_{u b} .
\end{gathered}
$$

Once the former linear equations have been solved, we can calculate the corresponding $v_{i}$ values in function of the selected $\Delta \rho_{j}$. Then, we take the value $e_{j}^{2}$ defined by

$$
e_{j}^{2}=\mathbf{v}^{T} \mathbf{Q}_{D}^{\geq 1} \mathbf{v}+\rho f^{2} \mathbf{m}^{T} \mathbf{Q}_{M}^{\geq 1} \mathbf{m}
$$

as the parameter for the suitability of $j$ th prism and the density possibility (negative or positive) adopted. So, in this $k$ th step, the method tests each of the unchanged prisms and both (negative and positive) density contrast possibilities. Then, the $j$ th prism and selected density (positive and negative) which produce a minimum value of $e_{j}^{2}$, which we will call $E(k)$, are definitively selected to increase the model, adding their effect to the model values $g_{i}^{c}$.

This process is successively repeated. For the successive steps, the scale value $f$ decreases and the trend parameters $p_{0}, p_{x}, p_{y}$ reach nearly stable values. The process stops when $f$ approaches 1 , resulting in some anomalous volumes and a final regional trend.

The $\rho$ parameter governs the application of the minimization conditions for the total anomalous mass and for the residual values. For low $\rho$ values, a better fit is obtained, but the anomalous mass may increase excessively (by means of volume increment) and become deformed, adopting a too deep position. For high $\rho$ values, the adjusted model can be too light and shallow.
On other hand, we observe that, through the model growth, the successive $E(k)$ values decrease continuously. But, for $f$ values very close to 1 , and especially for low $\rho$ values, the $E$ values can increase, giving rise to an inadequate mass increase (obtained with deep alternatively negative and positive near prisms). To avoid this effect, we impose an additional condition about the increased/decreased prism selection: for every step of the model growth, the $E$ value must be less than for the former step. This condition gives rise to minimum weighted mass models.

By adopting this last additional condition, we can establish a criterion for determination of the optimal $\rho$ value: we select, among the different $\rho$ values corresponding to minimum mass models, the $\rho$ value which produces a model with maximum total anomalous mass (see simulation example). According to equation (2), this criterion roughly corresponds to diminishing the residual gravity values $\mathbf{v}$. In fact, for higher $\rho$ values, the fit is rather poor, and the adjusted model is rather small and shallow, whereas for lower $\rho$ values, the last additional condition prevents an inadequate mass increase, and the growth process stops prematurely (see Figure 1).

\section{SIMULATION TEST RESULTS AND REAL DATA APPLICATION}

Figure 2 displays a simple simulation example. We consider a simple test model formed by a "+" body of positive density contrast $0.5 \mathrm{~g} / \mathrm{cm}^{3}$ and south-north orientation, near a " $\geq$ " body of negative density contrast $\geq 0.4 \mathrm{~g} / \mathrm{cm}^{3}$ and west-east orientation. The size of the " $\geq$ " body is $1100 \geq 500 \geq 400 \mathrm{~m}$ ( $x, y$, and $z$ coordinates, respectively), and the depth of its mass center is $328 \mathrm{~m}$. The "+" body has similar dimensions and a mean depth of $528 \mathrm{~m}$ (Figure 2). The anomalous gravity field is complemented by an arbitrary regional trend as $p_{0}+\left(x \geq x_{M}\right) p_{x}+\left(y \geq y_{M}\right) p_{y}$, where $p_{0}=25.00 \mathrm{mGal}, p_{x}=0.40 \mathrm{mGal} / \mathrm{km}$, and $p_{y}=$ $\geq 0.80 \mathrm{mGal} / \mathrm{km}$. A nonplanar regular distribution (stepped $100 \mathrm{~m}$ and with a approximate side of $3 \mathrm{~km}$ ) of simulated stations is adopted. The maximum and minimum heights of the gravity stations are $272 \mathrm{~m}$ and $66 \mathrm{~m}$, respectively. The simulated gravity anomaly has the simple appearance shown in Figure 3.

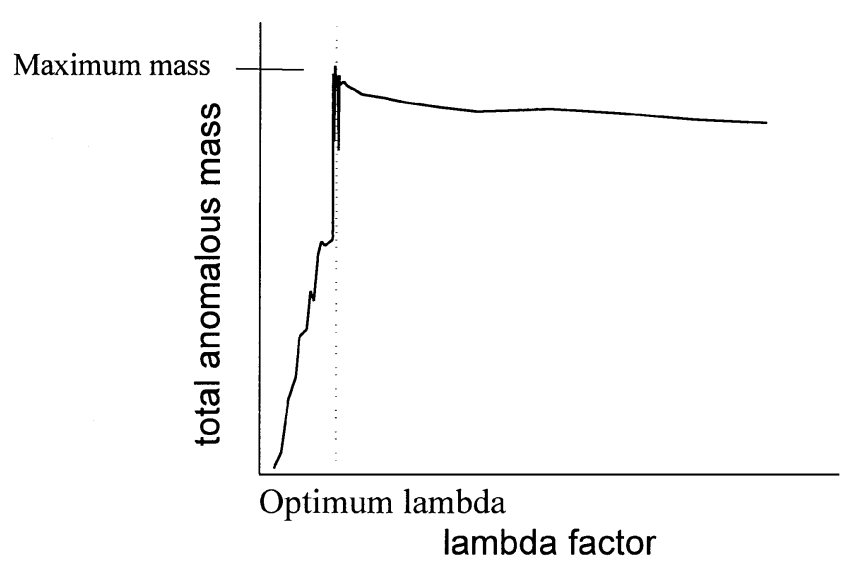

FIG. 1. Location of the optimum $\rho$ value as the value of the greatest anomalous mass. For experiments with a lower $\rho$ value, the growth process aborts prematurely, giving rise to an undeveloped model; for experiments with a higher $\rho$ value, a smaller anomalous mass is obtained. 
Moreover, we suppose the same accuracy for all of the gravity data.

We consider a volume under the survey area, reaching a maximum depth of $1200 \mathrm{~m}$, and we break it down into prisms with $100-m$ sides. An empty initial model is considered, namely, the case of no particular previous information. Then, we directly apply the aforementioned method to look for a regional trend and an adjusted anomalous volume for a prescribed density contrast of $0.5 \mathrm{~g} / \mathrm{cm}^{3}$ and $\geq 0.4 \mathrm{~g} / \mathrm{cm}^{3}$ everywhere. Through the matter expansion, the unknown parameters $p_{0}$, $p_{x}, p_{y}$, and $f$ show the evolution displayed in Figure 4. The final solution obtained gives a regional trend with the parameters $p_{0}=25.06 \mathrm{mGal}, p_{x}=0.43 \mathrm{mGal} / \mathrm{km}$, and $p_{y}=\geq 0.77$ $\mathrm{mGal} / \mathrm{km}$. Figure 5 shows the adjusted anomalous volumes, which result in a gravity adjustment with a root-mean-square error of $0.021 \mathrm{mGal}$.

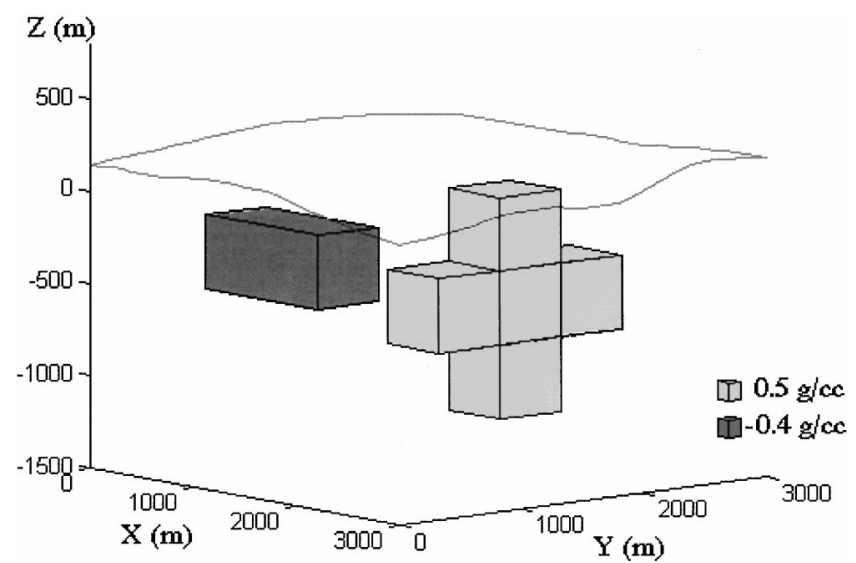

FIG. 2. Perspective of the simulation model formed by a south-north body ("+") of positive density contrast $0.5 \mathrm{~g} / \mathrm{cm}^{3}$ close to a west-east body (" $\geq$ ") of negative density contrast $\geq 0.4 \mathrm{~g} / \mathrm{cm}^{3}$.

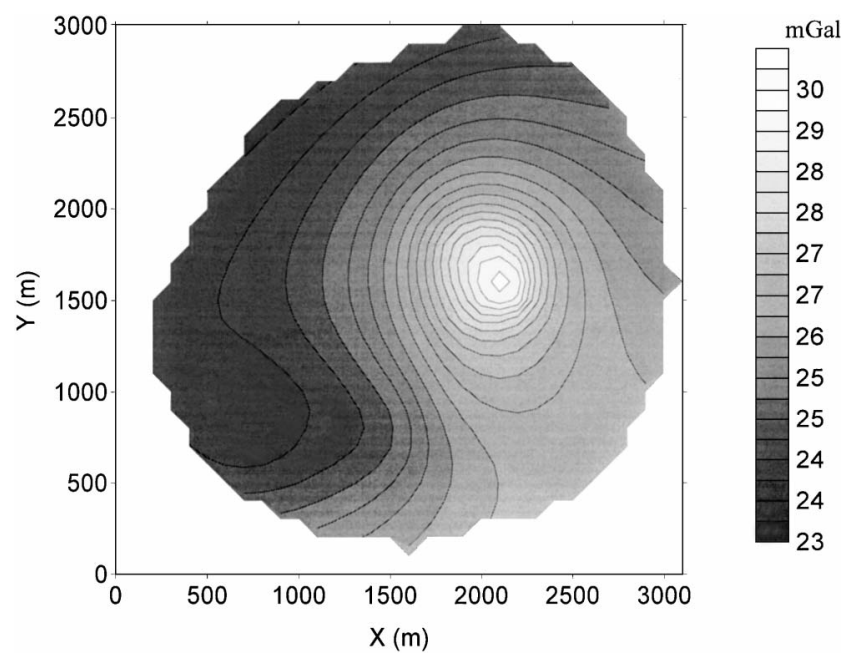

FIG. 3. Simulated gravity field of the model represented by Figure 2 plus a simulated linear regional trend. Contour interval of $0.5 \mathrm{mGal}$.
The morphology of the solution differs slightly from the supposed simulation model. Indeed, the additional condition of minimum anomalous mass, combined with the condition of minimum residual, gives rise to models which, for a level of fitness, look for compact volumes. So, the crossing region of the "+" body is thicker whereas the horizontal arms of the "+" body are thin and the downward arm is flat. Moreover, the effect of a small part of the anomalous masses (especially for the deepest region) is modeled by modifying the regional trend, with an anomalous mass economy. Nevertheless, the position
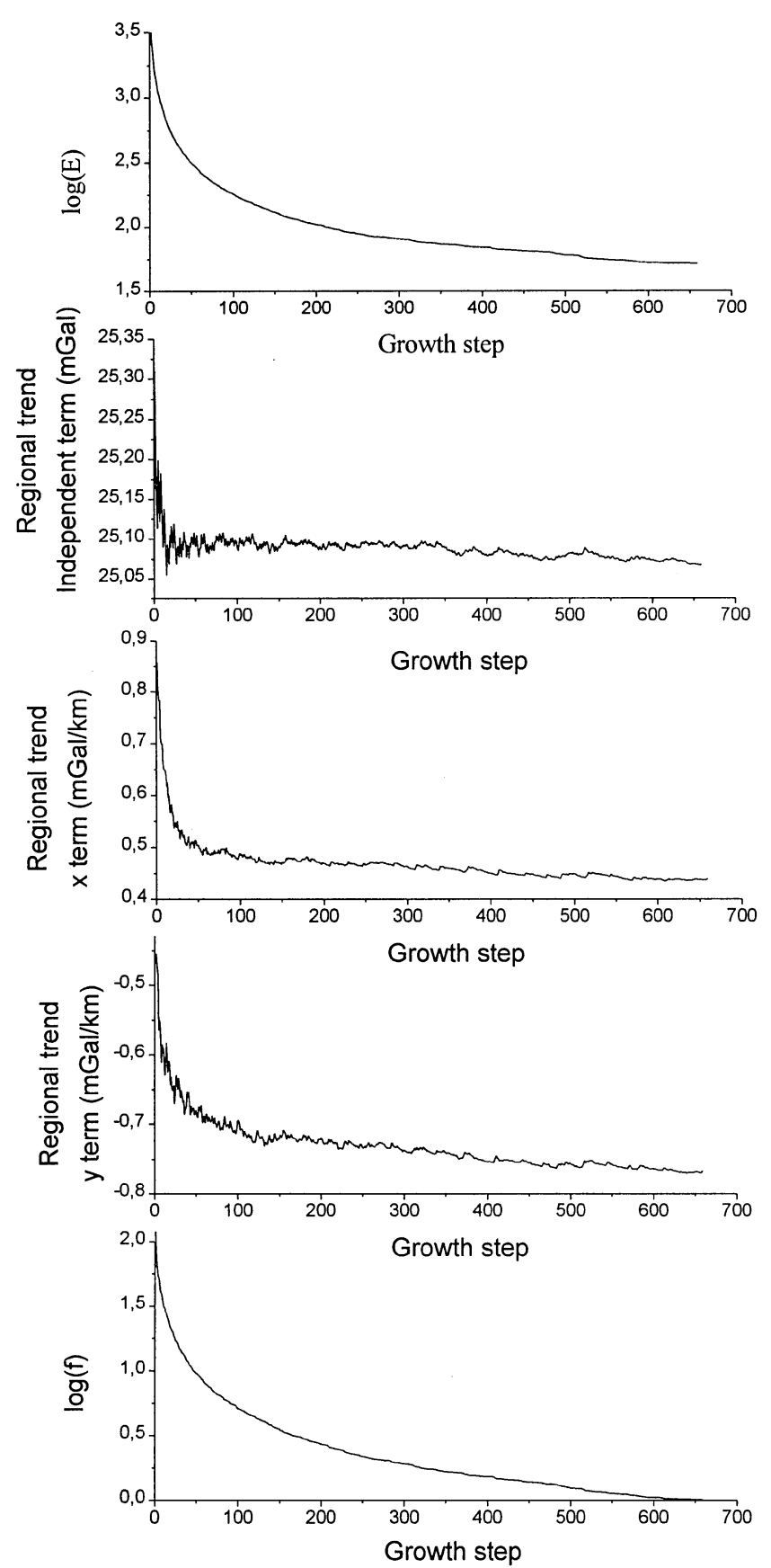

FIG. 4. Evolution of the fit parameter $E$ and the unknown parameters $p_{0}, p_{x}, p_{y}$, and $f$ through the step-by-step process. 
and general aspect of the solution is similar to the simulation model, showing the method is efficient.

To show the applicability of this method, we now consider a real example of a small survey on a volcanic island. The Canary Islands archipelago is located between $27^{\geq} 27^{\prime}$ and $29^{\geq} \geq 24^{\prime}$ north latitude and $18^{\geq} 10^{\prime}$ and $13^{\geq} 37^{\prime}$ west longitude, close to the African passive continental margin. This group of volcanic islands consist of seven main islands and several islets.

Gran Canaria is in the center of the archipelago and is the third largest island in surface area $\left(1540 \mathrm{~km}^{2}\right)$. It is formed by an almost conic volcanic edifice, covered by a network of ravines. More than the $75 \%$ of the total volume of the island was formed some 13-14 million years ago by the rapid extrusion of enormous amounts of basalts from fissural eruptions, probably making a flat-topped edifice (Araña and Carracedo, 1978). Nevertheless, eruptions have continued until recent times, including several episodes of explosive volcanism with the eruption of large-volume pyroclastic material associated with the formation of collapse calderas (Perez-Torrado et al., 1997). The subaerial evolution of the Gran Canaria volcanism can be divided into three main magmatic episodes (Anguita et al., 1991): first (14.5-8.5 Ma), buildup of a basaltic shield volcano probably located off the west coast and that led to a collapse caldera $15-20 \mathrm{~km}$ in diameter (Tejera caldera); second (5.5-2.7 Ma), the Roque Nublo cycle of eruption of materials apparently from a central vent near the center of the present island; and third (2.9 Ma to present), eruptions from small vents on the center and the northeast of the island.

Bosshard and MacFarlane (1970) performed a gravity study for the Western Canary Islands. They analyzed the characteristic positive anomaly of the main islands supposing a basement consisting of upwarped blocks. High-density bodies appear to rise from the elevated basement as volcanic plugs. For Gran Canaria, they interpreted a northwest-southeast characteristic profile of the Bouguer anomaly: a basement material with density $3.1 \mathrm{~g} / \mathrm{cm}^{3}$ appears elevated from a level of $10000 \mathrm{~m}$ depth to a top of $3000 \mathrm{~m}$ depth as a double plug, covered by three crustal layers of densities $2.7,2.6$, and $2.3 \mathrm{~g} / \mathrm{cm}^{3}$.

We consider here a gravity survey composed of 81 stations observed by the Spanish Geographical Institute, plus 17 stations observed by our own institution (Figure 6). Once the observations had been reduced and homogenized (to the IGSN71

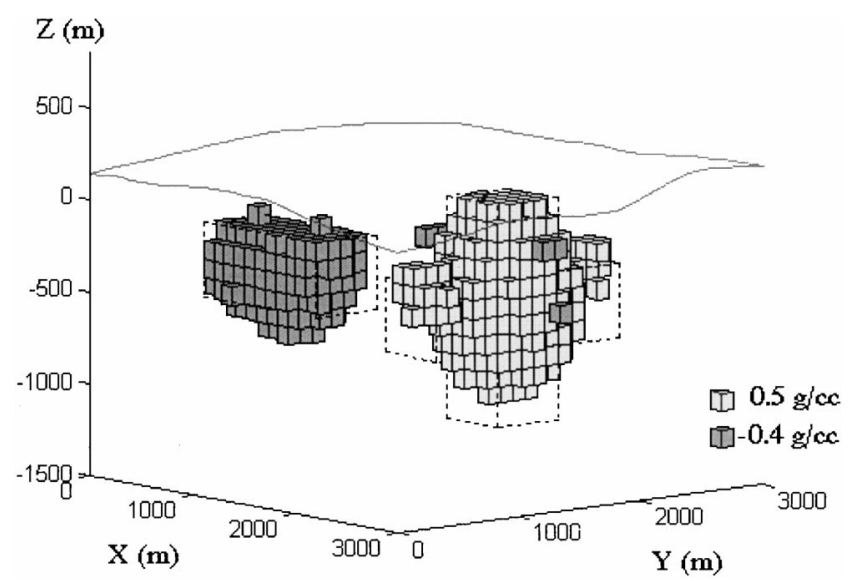

FIG. 5. Perspective of the anomalous model adjusted by the described growing method. standard), we calculated the Bouguer anomaly including a terrain correction that was determined using the terrain model shown in Figure 7. A value of $2.3 \mathrm{~g} / \mathrm{cm}^{3}$, as proposed by Bosshard and MacFarlane (1970), was used for the terrain and Bouguer mass correction. Figure 8 shows the map of the obtained Bouguer anomaly.

Taking into account the suspected high observational noise and the damaging effects of the very rugged topography, we applied a covariance analysis and a least-squares prediction (see Moritz, 1980; Camacho et al., 1997) to filter the noise and to interpolate a continuous anomaly field. Figure 9 shows the map of the interpolated Bouguer anomaly field; Figure 6 contains a contour map of the anomaly prediction error. Note that the determined anomaly field has an uncertainty of about 2 or $3 \mathrm{mGal}$.

Starting from the Bouguer anomaly field model shown in Figure 9 , we directly apply the proposed method of gravity inversion. To do so, a subsurface volume partition with

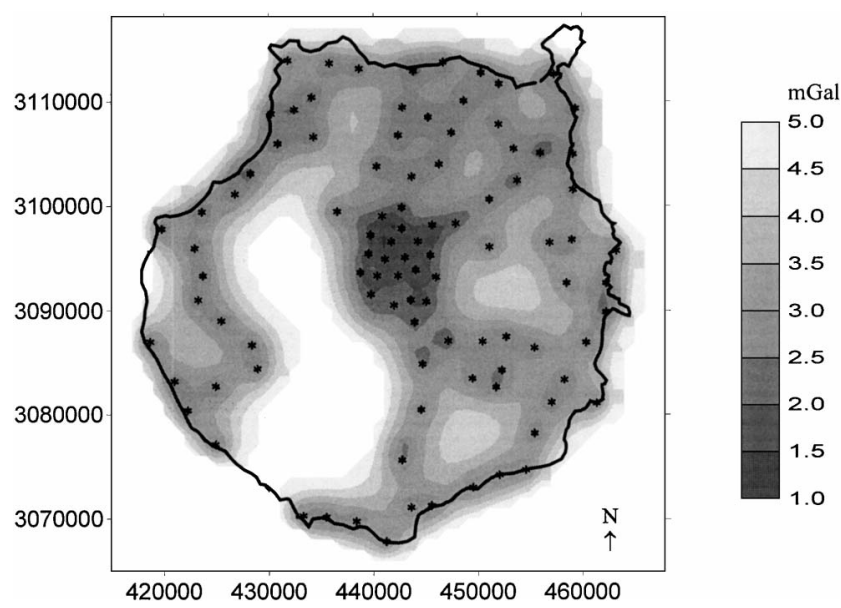

FIG. 6. Gran Canaria, Canary Islands, location of gravity stations and map of the Bouguer anomaly prediction error. Axis coordinates on UTM system $(\mathrm{m})$. The contour of the island is added in this figure and Figure 7.

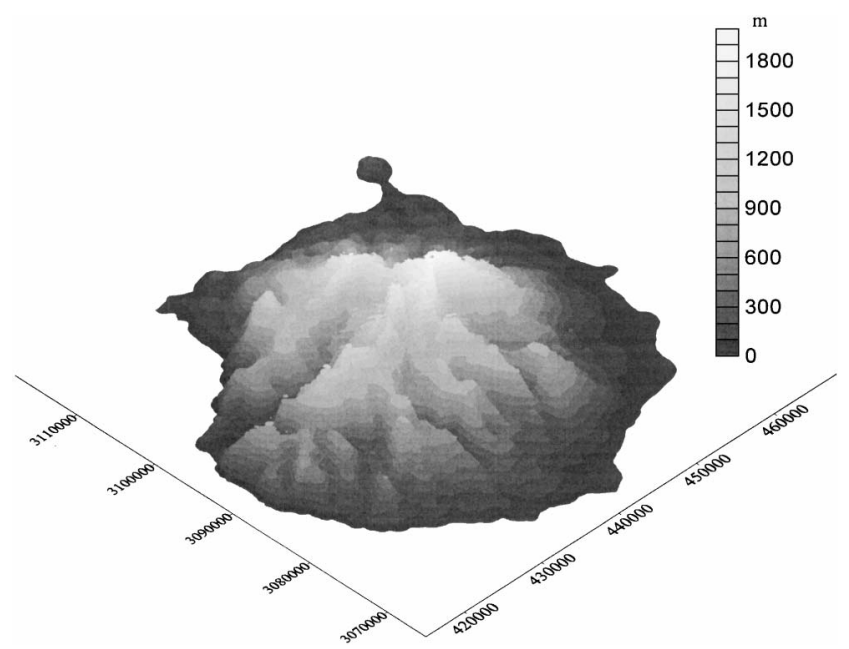

FIG. 7. Digital terrain model of Gran Canaria, Canary Islands, obtained by means of 1:50000 topographic chart digitalization. The highest elevations correspond to $1918 \mathrm{~m}$. 
parallelepipedic blocks of sides ranging from $1600 \mathrm{~m}$ (up blocks) to $3300 \mathrm{~m}$ (down blocks) was adopted. Taking into account the model densities proposed by Bosshard and MacFarlane (1970) and after several empirical trials, we selected the values $\geq 0.2 \mathrm{~g} / \mathrm{cm}^{3}$ and $0.3 \mathrm{~g} / \mathrm{cm}^{3}$ as the negative and positive contrast densities, respectively, for the model. The direct application of the described inversion method allowed us to obtain a contrast density model shown in Figure 10. Moreover, the inversion approach determined a regional trend (linear in our case) shown in Figure 11.

This regional component (Figure 11) is the northwestsoutheast trend associated with the main structural direction for the island. This trend, which is present in other islands of the archipelago, can be related to the change of the average crustal thickness and with the neighborhood of the continental margin.

The adjusted local model of density contrast is shown by some horizontal sections and vertical profiles (Figure 10). Several interesting features can be highlighted:

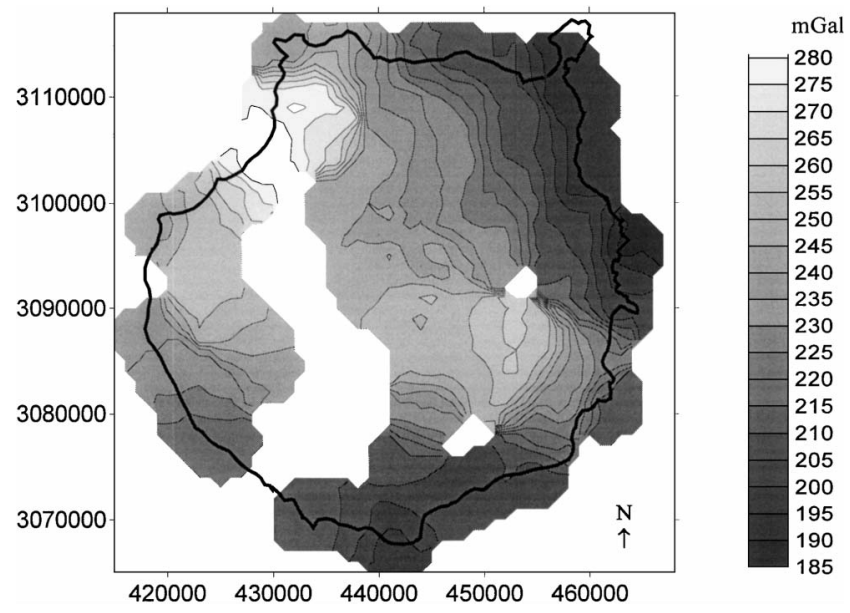

FIG. 8. Map of the observed Bouguer anomaly. Contour interval: $5 \mathrm{mGal}$. The white areas correspond to nonobserved zones.

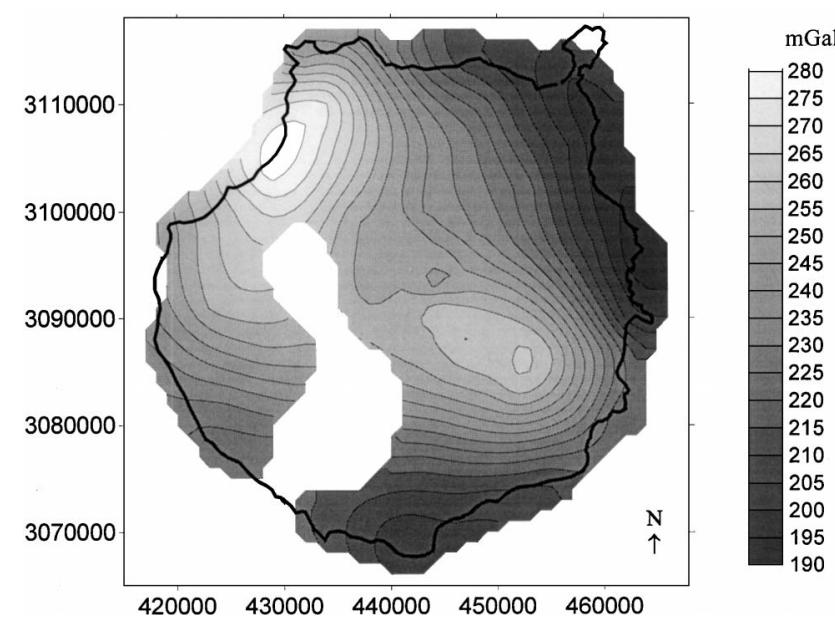

FIG. 9. Map of the Bouguer anomaly model, filtered and interpolated by least-squares prediction. Contour interval: $5 \mathrm{mGal}$.
1) The deep structure (horizontal profile, $17000-m$ depth) may be related to the ancient fissural extrusion of massive basaltic material that forms the main structure of the island as a volcanic plug. This high density body has a northwest-southeast alignment and is divided into two main structures (northwest and southeast), which are almost $3000 \mathrm{~m}$ deep at the top, near the areas of the ancient basaltic series (northwest border and, mainly, the southeast sector of the island).

2) A local minimum that can be associated to the ancient multicyclic stratovolcano and which, especially for rather superficial sections, just breaks the main fissural maximum, appears near the center of the island. This central vent, which is similar to the Teide volcano in Tenerife, included several episodes of explosive volcanism with the formation of collapse calderas.

3) Finally, rather linear lateral minima are to be seen in the adjusted model. These structures, located at shallow and medium depths, form two lines nearly parallel to the main northwest-southeast direction along the southwest and northeast sectors of the island and a third line southwestnortheast outside the northwest border. We relate these minima to a filling of light volcanic material in zones of fissures associated with the block displacements and the island volume expansion. Some recent volcanism can be associated with these minimum areas.

\section{CONCLUSIONS}

As main advantages of the method, we can point out the possibility of using positive and negative contrast simultaneously, the possibility of simultaneous determination of a simple regional trend, good geometrical results, the possibility of incorporating a previous (even inexact) model or working automatically without previous information, and the acceptance of inexact data. Nevertheless, as usual for the inverse gravimetric problem, the obtained solution must be accepted as a model (with a tendency to rather smooth shapes) whose validity is conditioned by the certainty of the adopted hypothesis. Moreover, the obtained model represents a distribution of anomalous masses up to an arbitrary horizontal stratification. With these usual restrictions, the proposed inversion method provides interesting 3-D information about the location, shape, and relative size of the anomalous masses (of prescribed density contrast) and in a nearly automatic approach without subjective contributions.

\section{ACKNOWLEDGMENTS}

We thank the reviewers for their very constructive comments on this paper.

\section{REFERENCES}

Al-Chalabi, M., 1971, Some studies relating to nonuniqueness in gravity and magnetic inverse problem: Geophysics, 36, 835-855.

Anguita, F., Garcia Cacho, L., Colombo, F., Camacho, A.G., and Vieira, R., 1991, Roque Nublo Caldera: A new stratocone caldera in Gran Canaria, Canary Islands: J. Volcanol. Geotherm. Res., 47, 45-63.

Araña, V., and Carracedo, J. C., 1978, Los volcanes de las Islas Canarias. III Gran Canaria: Ed. Rueda.

Barbosa, V. C. F., Silva, J. B. C., and Medeiros, W. E., 1997. Gravity inversion of basements relief using approximate equality constraints on depths: Geophysics, 62, 1745-1757. 

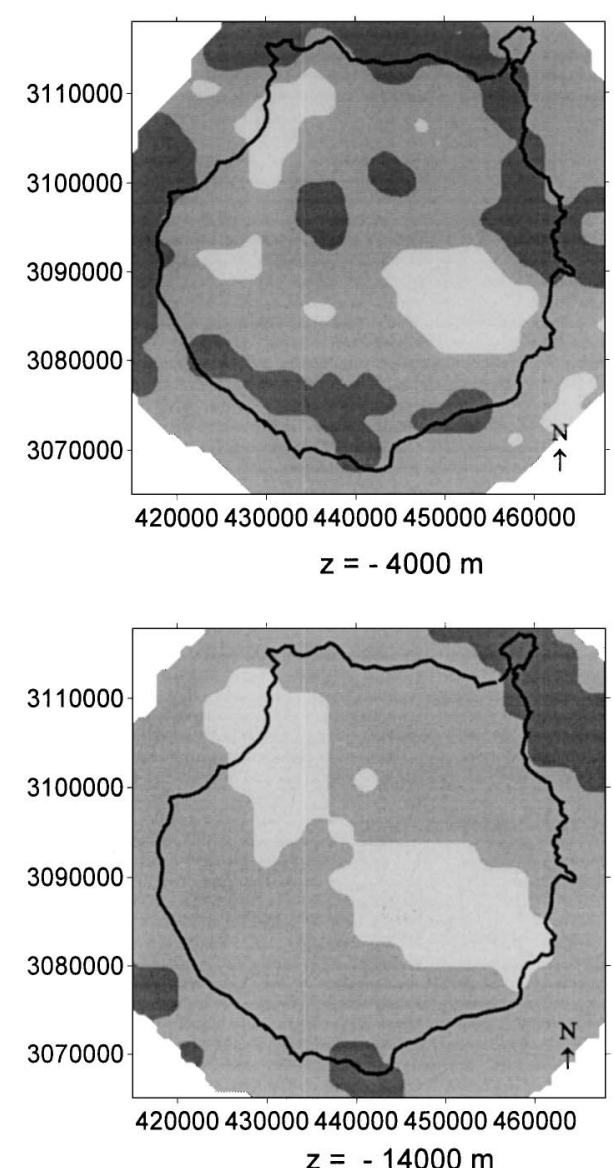

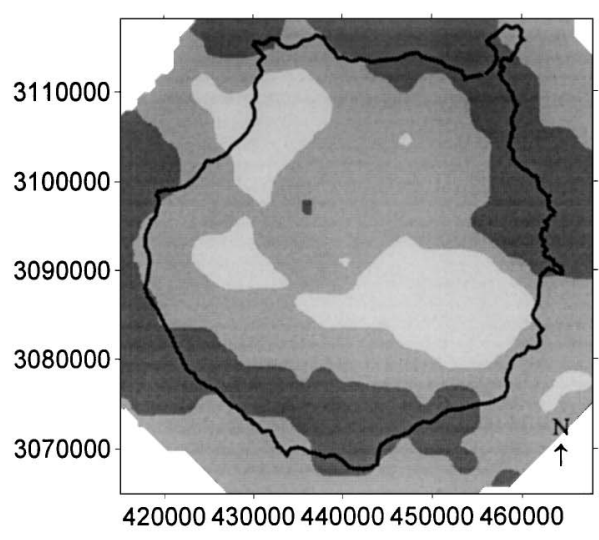

$z=-6000 m$

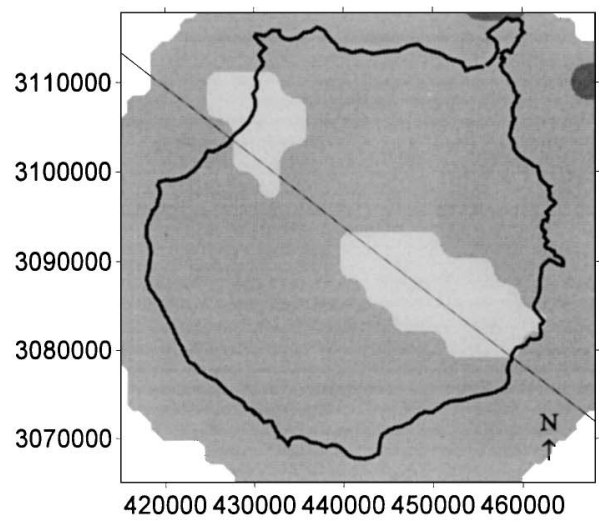

$z=-17000 m$
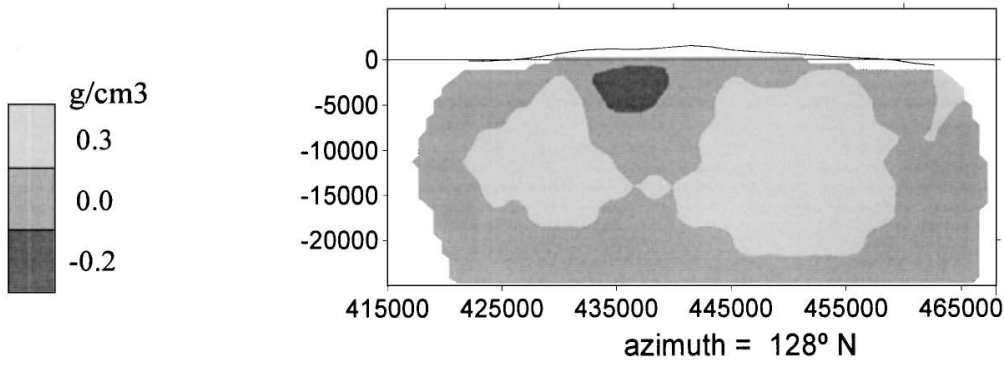

FIG. 10. Horizontal profile and vertical oblique section of the adjusted anomalous mass model.

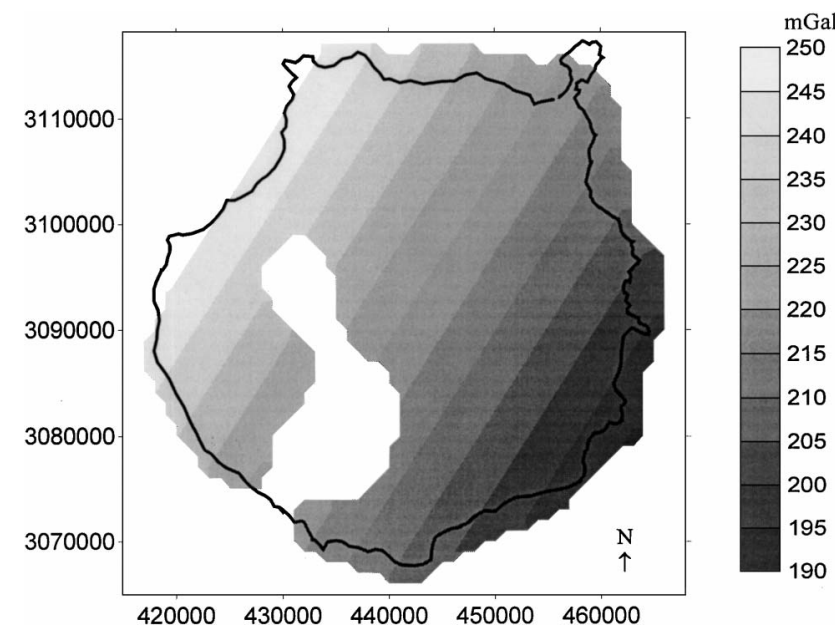

FIG. 11. Adjusted linear regional trend. The northwest- southeast trend is usual in the Canary Islands. Contour interval: $5 \mathrm{mGal}$.
Bosshard, E., and MacFarlane, D.J., 1970, Crustal structure of the Western Canary Islands from seismic refraction and gravity data: J. Geophys. Res., 75, 4901-4918.

Camacho, A. G., Montesinos, F. G., and Vieira, R., 1997, A threedimensional gravity inversion applied to Sao Miguel Island (Azores): J. Geophys. Res., 102, 7705-7715.

Moritz, H., 1980, Advanced physical geodesy: Herbert Wichmann Verlag.

Pedersen, L. B., 1979, Constrained inversion of potential field data: Geophys. Prosp., 27, 726-748.

Perez-Torrado, F.J., Marti, J., Mangas, J., and Day, S., 1997, Ignimbrites of the Roque Nublo group, Gran Canaria, Canary Islands: Bull. Volcanol., 58, 647-654.

Pick, M., Picha, J., and Vyskôcil, V., 1973, Theory of the Earth's gravity field: Elsevier Science Publ. Co.

René, R. M., 1986, Gravity inversion using open, reject, and "shapeof-anomaly" fill criteria: Geophysics, 51, 988-994.

Rothman, D. H., 1985, Nonlinear inversion, statistical mechanics, and residual statics estimation: Geophysics, 50, 2784-2796.

Silva, J. B. C. and Hohmann, G. W., 1983, Nonlinear magnetic inversion using a random search method: Geophysics, 46, 1645-1658.

Tarantola, A., 1988, The inverse problem theory: Methods for data fitting and model parameter estimation: Elsevier Science Publ. Co.

Zidarov, D., 1990, Inverse gravimetric problem in geoprospecting and geodesy: Elsevier Science Publ. Co. 